\title{
Hand Functions of Myoelectric and 3D-Printed Pressure-Sensored Prosthetics: A Comparative Study
}

\author{
Kyu Ho Lee, MD, MS ${ }^{1}$, Hobeom Bin, BS², KeunBae Kim, MD $^{3}$, \\ So Young Ahn, MD, PhD ${ }^{1}$, Bong-Ok Kim, MD, $\mathrm{PhD}^{1}$, Soo-Kyung Bok, $\mathrm{MD}$, $\mathrm{PhD}^{1}$
}

${ }^{1}$ Department of Rehabilitation Medicine, Chungnam National University Hospital, Chungnam National University School of Medicine, Daejeon; ${ }^{2}$ Department of Prosthetics and Orthotics, Chungnam National University Hospital, Daejeon; ${ }^{3}$ Chief Technology Officer, FunMove, Busan, Korea

\begin{abstract}
The loss of an upper limb significantly limits the functional activities of daily living. A huge emphasis is placed on the manipulation, shape, weight, and comfort of a prosthesis, to enable its use as an inherent body part. Even with technological advances, customized upper-extremity myoelectric prosthesis remain heavy and expensive. The high cost of upper-extremity prosthesis is an especially steep economic barrier for patients. Three-dimensional (3D) printing is a promising avenue for reducing the cost of prosthesis. We applied 3D-printed pressure-sensored prosthetics to a traumatic transradial amputee, and compared the hand functions with a customized myoelectric prosthesis. The 3D-printed pressure-sensored prosthetics showed low grip strength and decreased dexterity compared to the conventional myoelectric prosthesis. Although there were a few limitations, the fabrication of prosthesis with 3D printing technology can overcome previous problems such as high production cost, long fabrication period and heavy weight.
\end{abstract}

Keywords Prosthesis fitting, Three-dimensional printing, Amputation

\section{INTRODUCTION}

The extension of the average lifespan and resultant increase in peripheral vascular diseases and diabetes Korea has seen an increase in the frequency of amputations over the last several decades. Compared to lower-ex- tremities, upper-extremity amputations are less frequent. According to Kim et al., the ratio of upper-extremity to lower-extremity amputations is 1:2.2 [1]. Patients with upper-extremity amputations suffer from frustration and difficulty during the rehabilitation process, and experience paresthesia due to the loss of delicate movement

Received October 19, 2016; Accepted January 16, 2017

Corresponding author: Soo-Kyung Bok

Department of Rehabilitation Medicine, Chungnam National University Hospital, 266 Munhwa-ro, Chung-gu, Daejeon 35015, Korea. Tel: +82-42-3382460, Fax: +82-42-338-2461, E-mail: skbok@cnuh.co.kr

ORCID: Kyu Ho Lee (https://orcid.org/0000-0002-0136-8101); Hobeom Bin (https://orcid.org/0000-0003-4612-5856); KeunBae Kim (https://orcid. org/0000-0002-5374-3962); So Young Ahn (https://orcid.org/0000-0002-7447-0617); Bong-Ok Kim (https://orcid.org/0000-0002-4831-5023); SooKyung Bok (https://orcid.org/0000-0002-8957-2827).

(c) This is an open-access article distributed under the terms of the Creative Commons Attribution Non-Commercial License (http://creativecommons.org/ licenses/by-nc/4.0) which permits unrestricted noncommercial use, distribution, and reproduction in any medium, provided the original work is properly cited. Copyright $\odot 2017$ by Korean Academy of Rehabilitation Medicine 
by the hands, complicated tactile senses of the upper extremities, and proprioceptive sensory functions [2]. Although numerous upper-extremity prosthetics are being developed for upper-extremity amputees, economical high-level prosthetics that satisfy the functions of individual patients are still difficult to obtain [2].

Advanced myoelectric prosthesis are currently being developed that express the myoelectric signals of muscle contractions into functions desired by users, through the use of surface electrodes. Although this method uses a 'myoelectric control system' to control the myoelectric prosthesis, fabricating this system incurs a tremendous cost and enormous amount of time [3]. In addition, other problems persist, such as heavy weight, impracticable fine motor control, and slow movements.

Unlike lower-limb amputees, many unilateral upperlimb amputation patients do not use prosthesis since they are capable of conducting activities of daily living (ADL) with the remaining healthy upper limb. In addition, since upper-extremity prosthesis are relatively exposed compared to lower-extremity prosthesis, patients regard the manipulation, shape, and convenience as important issues when fitting a prosthesis. Thus, the majority of amputees only use their prosthesis for cosmetic reasons, and hence their requirement for easily accessibility and light weight.

Even with advances in technologies, the heavy weight and high expense of customized upper-extremity prosthesis remain stumbling blocks that discourage upperextremity amputees from appraising myoelectric prosthesis. The cost of a myoelectric prosthetic hand ranges from USD 9,000 to even 40,000; depending on the mode of control and amputation of forearm, these devices require extensive fitting procedures to develop the final device, and often includes a complex system of cables and harnesses. The new technique of three-dimensional (3D) printing has recently been applied to various areas in the manufacturing and medical industry, and its development has now reached the stage of fabricating tailored medical devices. The application of 3D printing technology in manufacturing prosthesis has the potential to simplify the process and easily be applied to the medical industry. Recent technology has advanced to the stage of applying 3D scanning, computer-aided design (CAD), and computer-aided manufacturing (CAM) to the fabrication of customized prosthesis. Recent CAM and
3D printing technology can fabricate prosthesis suitable to actual patients, with their precise measurements and excellent fabrication capabilities [4]. The development of CAD programs, manufacturing materials, and opensource image editing software has made the development of prosthetic hands and other assistive devices possible [4]. Such 3D-printed prosthesis are hot topics of conversation in the mass. Thus, this paper reports an assessment of the comparison in hand functions of an existing customized myoelectric prosthesis for upper-extremity amputees and a newly fitted 3D-printed pressure-sensored prosthesis (3D-PSP).

\section{CASE REPORT}

A 52-year-old male patient had a crushed right forearm due to an accident involving a washing machine. He was admitted to the emergency department of Chungnam National University Hospital. A right transradial amputation was performed as an emergency surgery. After postoperative wound care, he was transferred to the Department of Rehabilitation Medicine for comprehensive rehabilitation and prosthesis-fitting. On admission, the patient was put on medication to stabilize the operated site and control the pain of the site, phantom pain, and sleep disturbance. The power and range of motion of the elbow and shoulder at the amputation side were of good grade, as assessed by the manual muscle test. In order to enhance the muscle strength until fabrication of the prosthesis, exercises for elbow flexor strengthening and stump supination and pronation were performed. In addition, after being educated on the overall exercise to enhance the muscle strength of the upper extremity with a Thera-Band, the patient was instructed to perform the exercise by himself. Simple training for ADL, such as opening and closing a hook, supination, and pronation, was performed with a training prosthesis, which was a myoelectric prosthesis for demonstration of the palmar prehension grasp, similar to those fitted on the patient. The equipment owned by the assistive technology center of our hospital for patient training was rented and used. Since it was not a custom-made prosthesis, there was a limitation to the patient's ability to work smoothly, but to the main aim was to increase the sensor adaptability.

After stabilizing the patient's condition and considering his health, economic status, and opinion, we decided 
to fit a myoelectric prosthesis. Because the patient was young and economically unconstrained, he desired a myoelectric prosthesis due to its functional capability, although it was expensive. For a comparison, we fabricated two types of prosthesis; their characteristics are summarized in Table 1.

\section{Myoelectric prosthesis}

A customized myoelectric prosthesis (MP) (840 g) was fitted for functional use. Both MP and 3D-PSP were fitted at 2 months after the injury, when the wound site was stable and the volume of muscle was constant. The optimal locations of the electrodes were decided through biofeedback, using an electromyogram. The prosthetic hand (digital twin $8 \mathrm{E} 41=7$, Otto Bock) was operated with a palmar prehension grasp by using two myoelectric sensors to detect the electrical activities of the flexor carpi radialis and extensor carpi radialis (Fig. 1). A prosthetic hand was fabricated to realize a voluntary opening motion by the application of electric signals from movements of the extensor with an attached electrode, and to realize the voluntary closing with movement of the flexor. The prosthetic hand was fabricated from silicon in the shape of a cosmetic prosthesis, and the cost for the prosthesis fitting was USD 13,000.

Regular occupational therapy training was conducted for 30 minutes every day, while wearing the prosthesis. In addition, 30 minutes special training time every day was also arranged under the guidance of a rehabilitation doctor. The patient underwent ADL training and strengthening exercise of the amputated upper extremity under the guidance of a skilled occupational therapist while wearing the prosthesis. In the early stages, training such as stacking cones and moving cups with the prosthesis, fastening buttons and zippers with both hands, and bilateral coordination using threads was performed. After the patient became familiar with the use of the prosthesis to a certain degree, the ADL training was enhanced with more complicated and difficult functions, such as stacking cups, pouring water, and writing. The total training period lasted for 2 weeks.

\section{D-printed pressure-sensored prosthesis}

Using a 3D scanner (Artec Eva 3D Scanner; Artec, Santa Clara, CA, USA) and CAD software (Z Brush), we fabricated a 3D-PSP for comparison with the existing customized myoelectric prosthesis. Poly lactic acid was used to print the materials. Pressure sensor was used to detect the change in volume and pressure of the flexor carpi radialis during the voluntary palmar grasp. A prosthetic hand was fabricated to realize a voluntary closing motion by the application of pressure signals from movements of the flexor carpi radialis with the attached pressure sensor, and to realize a voluntary opening by releasing the muscle. The appearance of the 3D-PSP can be seen in Fig. 2. With an equipped 3D-printer and 3D-scanner, the estimated cost of 3D-PSP was USD 440. The training method and intensity were the same as with MP. After the hand function test and assessment of hand activities, only the MP training was performed for patient ADL functions.

Specifications and features of the existing myoelectric prosthesis and the newly fitted 3D-PSP were compared. In order to compare the hand functions of the two prostheses, a nine-hole pegboard test, box \& block test, and hand strength test were conducted. As mentioned earlier, each test was performed after training for 2 weeks. Differ-

Table 1. Comparison between myoelectric and 3D-printed pressure-sensored prosthesis

\begin{tabular}{lll}
\hline & \multicolumn{1}{c}{ Myoelectric prosthesis } & 3D-printed pressure-sensored prosthesis \\
\hline Prosthetic hand & Digital twin 8E41=7, Otto Bock & $\begin{array}{l}\text { Artec, Eva 3D Scanner, Z Brush } \\
\text { MakerBot Replicator Z18 3D Printer }\end{array}$ \\
$\begin{array}{ll}\text { Socket } \\
\text { Weight }(\mathrm{g})\end{array}$ & $\begin{array}{l}\text { Polypropylene plastic socket } \\
\text { Sensor }\end{array}$ & 600 \\
& $\begin{array}{l}\text { 2 myoelectric sensors } \\
\text { (Detect electrical activity of flexor carpi } \\
\text { radialis and extensor carpi radialis) }\end{array}$ & 1 pressure sensor \\
Battery & Li-ion $7.2 \mathrm{~V}(65 \mathrm{~g})$ & Li-ion 3.7 V (15 g) \\
Cost (USD) & 13,000 & 440 \\
Fabrication time & 2 weeks & 4 days \\
\hline
\end{tabular}




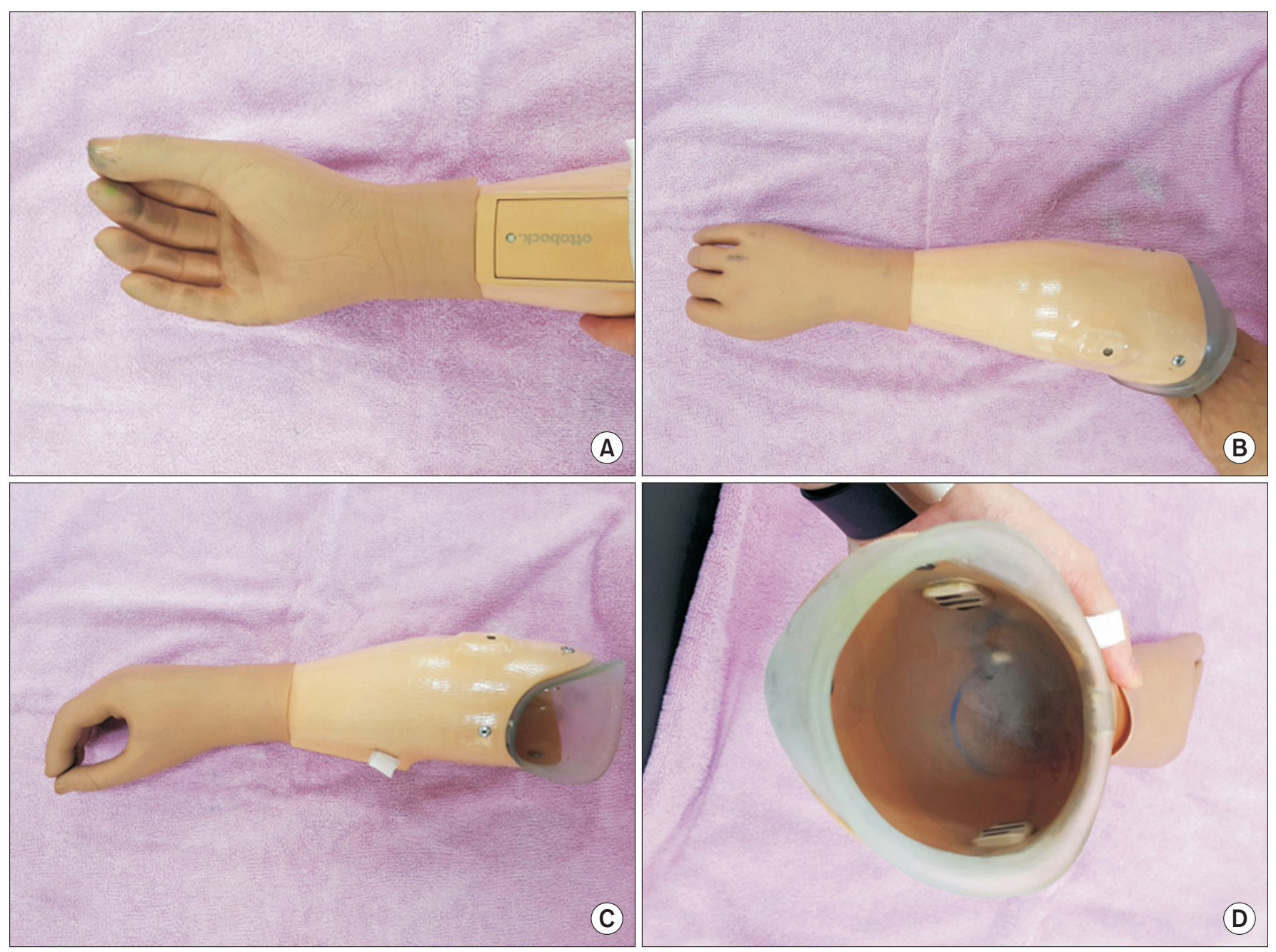

Fig. 1. Myoelectric prosthesis (MP). (A) Palmar aspect view of MP with attached battery. (B) Dorsum aspect view of MP. (C) Lateral view of MP. The prosthetic hand was operated with a palmar prehension grasp. (D) Socket view of MP. Two myoelectric sensors are attached to the inner side.

ent outcomes were observed with both prostheses. In each test, the patient performed better with the customized MP than with the 3D-PSP. In the nine-hole pegboard test, the patient took 82 seconds with the MP and 86 seconds with the 3D-PSP. In the box \& block test, the patient marked 19 blocks with the MP and 20 blocks with the 3D-PSP. In the grip strength test, the patient recorded $30 \mathrm{lbs}$ with the MP and $13 \mathrm{lbs}$ with the 3D-PSP (Table 2). We used equipment from 'Baseline $300 \mathrm{lb}$ Digital LCD Head Hydraulic Pinch Gauge' test to measure the maximum hand strength of each prosthesis. In the hand function test, the MP recorded 30 pounds (lbs) of pinch power (Table 2). This result was higher than the maximum grip power 90 newton $(\mathrm{N})$, presented by the Otto Bock company. This is considered due to a difference in the equipment used for the test, and the sensitivity difference of the equipment.

The hand activities were measured by self-performance in 5 activities: transfer the paper cup, dressing the button, wearing the socks, writing, and gripping the small corn. Each item was recorded as Yes or No. The assessment revealed a better performance by the patient with the customized MP than with the 3D-PSP. In the selfperformance of 5 activities, the patient accomplished all items with the MP, but only transfer the paper cup and wearing the socks items with the 3D-PSP (Table 3).

\section{DISCUSSION}

The present case compared a customized MP and a 3D-PSP. It is important to note that prosthesis made with 


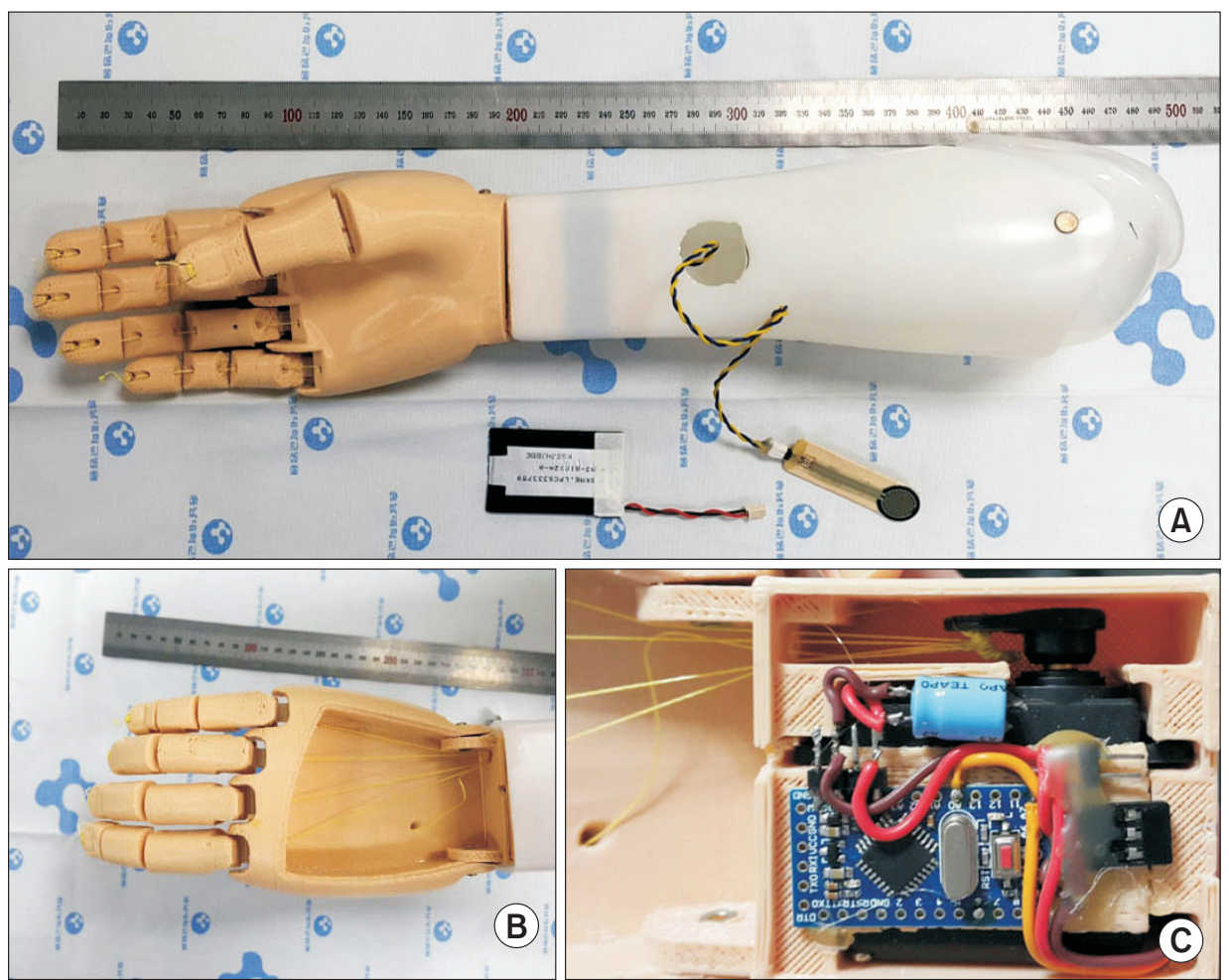

Fig. 2. A 3D-printed pressuresensored prosthesis (3D-PSP). (A) Palmar aspect view of 3D-PSP. It consists of a prosthetic hand (3Dprinted by poly lactic acid material), socket (polypropylene plastic socket), battery (Li-ion $3.7 \mathrm{~V}$ ), and a pressure sensor. (B) Dorsum aspect view of the prosthetic hand. The finger joints are connected by nylon fiber. (C) Motor driving box connected to the prosthetic hand at the wrist level, when the socket is detached.
Table 2. Hand function test of myoelectric and 3D-printed pressure-sensored prosthesis

\begin{tabular}{lcc}
\hline & $\begin{array}{c}\text { Myoelectric } \\
\text { prosthesis }\end{array}$ & $\begin{array}{c}\text { 3D-printed } \\
\text { pressure- } \\
\text { sensored } \\
\text { prosthesis }\end{array}$ \\
\hline Nine hole pegboard test (s) & 82 & 86 \\
Box \& Block test (blocks) & 19 & 20 \\
Hand strength (lbs) & 30 & 13 \\
\hline
\end{tabular}

a $3 \mathrm{D}$ printer is not widely used in the Korea. The 3DPSP showed lower grip strength and decreased dexterity compared to the conventional MP. Current 3D printing technology has several limitations to replace the existing prosthesis, which is produced by skilled technicians. However, it's numerous advantages include low production cost, short fabrication period, and light weight. It has the significant potential to positively impact the quality of life and daily usage for an uninsured or economically disadvantaged amputee, by allowing easy access to the electrical prosthesis. Also, the patient can easily print new devices as required, with a relatively low economic burden. In addition, if 3D printing technology is partially applied to existing prosthesis fabrication, not only high
Table 3. Assessments of hand activities in myoelectric and 3D-printed pressure-sensored prosthesis

\begin{tabular}{lcc}
\hline & $\begin{array}{c}\text { Myoeletric } \\
\text { prostehsis }\end{array}$ & $\begin{array}{c}\text { 3D printed } \\
\text { pressure- } \\
\text { sensored } \\
\text { prosthesis }\end{array}$ \\
\hline Transfer the paper cup & Yes & Yes \\
Dressing the button & Yes & No \\
Wearing the socks & Yes & Yes \\
Writing & Yes & No \\
Grip the small corn & Yes & No \\
\hline
\end{tabular}

The patient conducted each items is recorded 'Yes' and otherwise is 'No'.

quality of existing prosthesis but also a reduction in cost can be expected.

Such 3D printing technology is actively used for tailored production to reflect individual needs in manufacturing areas that are shifting to systems for producing small batches [5]. Furthermore, it is driving major innovations in many areas, such as engineering, art, education, and medicine. In medicine, 3D bio-printing is already being used for the generation and transplantation of several tissues, including multilayered skin, bone, vascular grafts, tracheal splints, heart tissue, and cartilaginous structures 
[5]. Products fabricated through 3D printing technology are sensitively responsive to individual characteristics and demands, have the potential for rapid prototyping, use materials that are easy to change, and can be made again using the early measurement data. Thus, the application of 3D printing technology to the fabrication of prosthesis for the rehabilitation of people with disabilities would overcome the problems of high cost and higher amount of time required for conventional prosthesis [6]. The use of 3D printing technology also increases the convenience for people with disabilities, in that it can quickly respond to the breakdown or damage of a device. Therefore, the aim of this study was to determine the current status, address the problems, and weigh the possibility of clinically universal prosthetic arms, by exploring a prosthesis fabricated by $3 \mathrm{D}$ printing technology and comparing it with an existing MP.

This design has few limitations. First, the short battery life and motor noise during the motion in 3D-PSP restricts the use of the prosthesis. Second, the patient was severely repulsed by the aesthetics. When a prosthesis is applied to a severed body part, patients tend to be reluctant to expose even a cosmetic prosthesis. In the case of 3D-PSP, the patient was repulsed by the continued use of the prosthesis because of the strong mechanical feeling. Third limitation was the low grip strength of the terminal device, and low durability of the mechanical components. The motors and parts of 3D-PSP fell short of the grip power provided by the MP, and the electronic equipment and parts of the prosthetic hand were easily damaged by shocks. The poly lactic acid (PLA) used to fabricate the prosthesis was an ecofriendly biodegradable resin made from sugarcane and corn with few toxic ingredients [7]. It is mainly used to produce prosthesis and is more applicable since it provides less contraction and leaves less residue than acrylonitrile butadiene styrene (ABS) during processing. However, it has very low strength and is susceptible to water. Fourth, with current 3D printing technology, it is difficult to fabricate the delicate socket parts in contact with the severed part of the user. Despite the precise measurement of the amputated site with 3D scanning, errors were encountered in the actual fabrication of the product, causing an inconvenience and ill-fit with the socket of the severed body part. Fifth, there are many differences between MP and 3D-PSP sensor mechanisms, which may have caused the differences in hand function test and assessment of hand activities. While MP was used for myoelectric sensor, the 3D-PSP used a relatively less sensitive pressure sensor. When the myoelectric sensor is attached to the 3DPSP, the volume of the prosthesis becomes huge and the manufacturing becomes complicated.

Even with these limitations, the fabrication of prosthesis with 3D printing technology can overcome the various problems and become an appropriate method to compensate for previous problems related to prosthesis. Our prosthetic device may have significant potential to positively impact the development of upper-extremity prosthesis fabrication. Further studies require examining the functionality, validity, durability, and benefits of this 3D-PSP.

\section{CONFLICT OF INTEREST}

No potential conflict of interest relevant to this article was reported.

\section{REFERENCES}

1. Kim YC, Park CI, Kim DY, Kim TS, Shin JC. Statistical analysis of amputations and trends in Korea. Prosthet Orthot Int 1996;20:88-95.

2. Jang CH, Yang HS, Yang HE, Lee SY, Kwon JW, Yun BD, et al. A survey on activities of daily living and occupations of upper extremity amputees. Ann Rehabil Med 2011;35:907-21.

3. Oskoei MA, Hu H. Myoelectric control systems: a survey. Biomed Signal Process Control 2007;2:275-94.

4. Dombroski CE, Balsdon ME, Froats A. The use of a low cost 3D scanning and printing tool in the manufacture of custom-made foot orthoses: a preliminary study. BMC Res Notes 2014;7:443.

5. Weller C, Kleer R, Piller FT. Economic implications of 3D printing: market structure models in light of additive manufacturing revisited. Int J Prod Econ 2015;164: 43-56.

6. Zuniga J, Katsavelis D, Peck J, Stollberg J, Petrykowski M, Carson A, et al. Cyborg beast: a low-cost 3d-printed prosthetic hand for children with upper-limb differences. BMC Res Notes 2015;8:10.

7. Lee JY, Tan WS, An J, Chua CK, Tang CY, Fane AG, et al. The potential to enhance membrane module design with 3D printing technology. J Memb Sci 2016;499: 480-90. 